\title{
Clear cell sarcoma originating in the anterior mediastinum
}

\author{
Katsunori Masaki $\cdot$ Katsuhiko Naoki $\cdot$ Kenzo Soejima $\cdot$ Koichi Sayama $\cdot$ Nobufumi Kamiishi \\ Kyuto Tanaka - Kota Ishioka - Aya Sasaki - Yuichiro Hayashi - Takeru Funakoshi - Michiro Susa • \\ Hideo Morioka $\cdot$ Sadatomo Tasaka $\cdot$ Koichiro Asano $\cdot$ Tomoko Betsuyaku
}

Received: 10 August 2012/Accepted: 26 February 2013/Published online: 15 March 2013

(C) The Japan Society of Clinical Oncology 2013

\begin{abstract}
A 34-year-old man with intermittent chest pain had a $60-\mathrm{mm}$ mass in the anterior mediastinum. A CTguided needle biopsy revealed that the tumor was composed of atypical small cells and was positive for HMB-45 without cytoplasmic melanin pigmentation. These results suggested a diagnosis of malignant melanoma (MM) or clear cell sarcoma (CCS). EWS gene rearrangement was detected by fluorescence in situ hybridization, resulting in the definite diagnosis of CCS. Sarcomas originating in the mediastinum account for only $1 \%$ of all sarcoma cases. CCS is a rare soft tissue sarcoma, which usually occurs in the tendons and aponeuroses and is often misdiagnosed as MM because of similar immunophenotypes. There is only one reported case of a primary CCS of the mediastinum. There is no standard treatment for CCS, but for metastatic MM, dacarbazine, ipilimumab, and BRAF-inhibitors can be treatments of choice. Thus, differential diagnoses are important to determine treatment strategies. Meticulous
\end{abstract}

K. Masaki · K. Naoki $(\bowtie) \cdot$ K. Soejima · K. Sayama .

N. Kamiishi - K. Tanaka - K. Ishioka - S. Tasaka - K. Asano ·

T. Betsuyaku

Division of Pulmonary Medicine, Department of Internal

Medicine, Keio University School of Medicine,

35 Shinanomachi, Shinjuku-ku, Tokyo 160-8582, Japan

e-mail: knaoki@pg7.so-net.ne.jp

A. Sasaki · Y. Hayashi

Department of Pathology, Keio University School of Medicine,

Tokyo, Japan

T. Funakoshi

Department of Dermatology, Keio University School

of Medicine, Tokyo, Japan

M. Susa $\cdot$ H. Morioka

Department of Orthopedic Surgery, Keio University School

of Medicine, Tokyo, Japan observation of skin lesions and pathological and molecular diagnoses, including EWS gene rearrangement, is recommended in cases with mediastinal tumors suspected of MM or CCS.

Keywords Clear cell sarcoma - Mediastinal tumor . EWS gene rearrangement - Malignant melanoma

\section{Introduction}

Recent advancements in genetic testing of malignant diseases have improved understanding of the diseases and treatment outcomes, such as with $E G F R$ mutation and $A L K$ rearrangement. Clear cell sarcoma (CCS) is a relatively rare disease and has a similar immunophenotype as malignant melanoma (MM), but with distinct molecular characteristics and treatment strategy. Here we report a rare case of mediastinal tumor finally diagnosed as CCS using genetic testing, i.e., fluorescence in situ hybridization (FISH).

\section{Case reports}

A 34-year-old male nonsmoker presented to the Keio University Hospital with intermittent chest pain that first occurred approximately 6 months previously during exercise. His anterior chest wall was painful to the touch. Upon physical examination, there were no specific findings or skin eruptions. Chest radiographs and computed tomography $(\mathrm{CT})$ revealed a $60 \times 36-\mathrm{mm}$ mass in the anterior mediastinum with sternal invasion (Fig. 1). Complete blood count revealed mild anemia (hemoglobin $10.4 \mathrm{~g} / \mathrm{dl}$ ), but was otherwise unremarkable. Serum tumor markers 

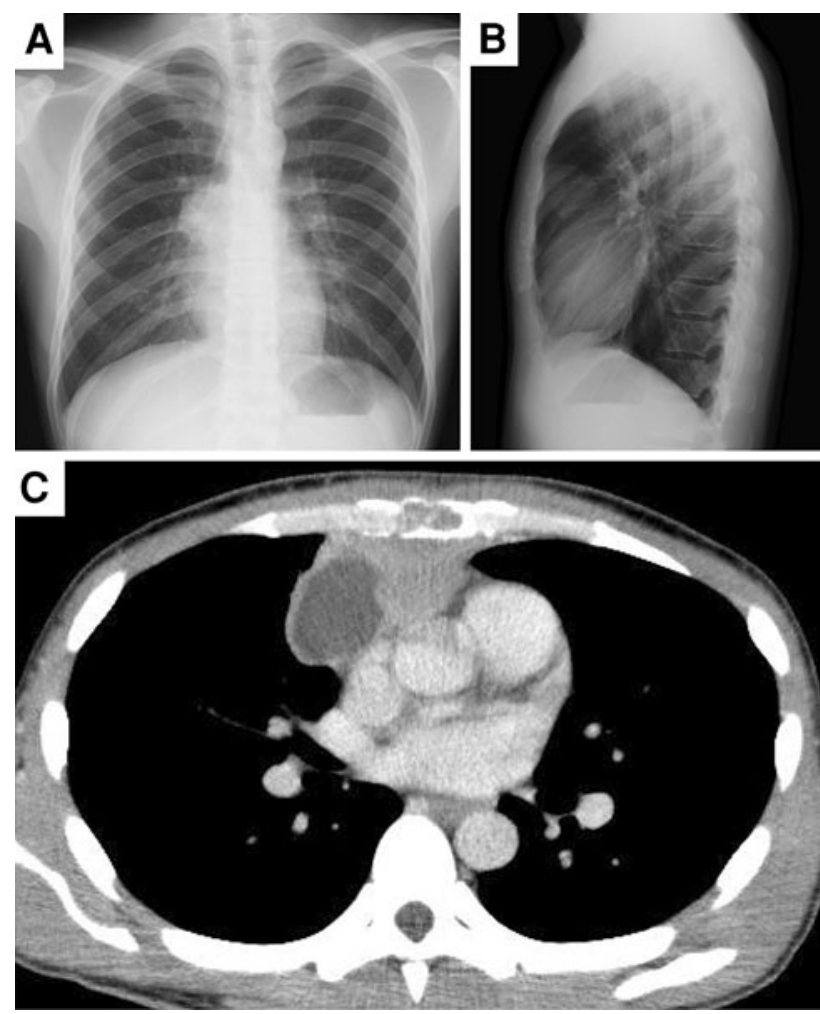

Fig. 1 An anterior mediastinal tumor revealed by chest radiograph (a, b) and computed tomography (c)

(carcinoembryonic antigen, progastrin releasing peptide, neuron specific enolase, alpha-fetoprotein, $\beta$-human chorionic gonadotropin, and soluble interleukin-2 receptor) were within normal limits.

A CT-guided needle biopsy was performed for pathological diagnosis. The major component of the tumor was atypical small cells with round-oval nuclei, prominent basophilic nucleoli, and plump fusiform cytoplasm by simple hematoxylin-eosin staining (HE staining) (Fig. 2a). The tumor was highly active mitotically (1-3 per high power field). Thus, it was a poorly differentiated carcinoma or high-grade sarcoma; pathological diagnosis was difficult in terms of HE staining. Ewing family of tumours, melanotic schwannoma, nevus, or chondroid chordoma was incompatible with histological findings, the lesion of the tumor, and his clinical course. On immunohistochemistry, the tumor was positive for HMB-45 (Fig. 2b) and S-100 protein (focal) and negative for Melan-A (Fig. 2c), widespectrum cytokeratin (Dako. Monoclonal Mouse AntiHuman Cytokeratin Clones AE1/AE3), $\alpha$-smooth muscle actin, desmin, CD5, CD34, and CD99, which was specific for primitive neuroectodermal tumour.

Based on the characteristics of the HMB-45 positive spindle cell sarcoma without cytoplasmic melanin pigmentation, MM or CCS was considered. Although angiomyolipoma is also stainable with HMB-45, it was not considered because of the lesion of tumor and the findings of HE staining. EWS gene rearrangement was detected by FISH using the EWS (22q12) dual-color, break-apart probe (Vysis, LSI EWSR1 Break Apart FISH Probe Kit), which spans the known common breakpoints in the EWS gene on chromosome 22. The $1100 \mathrm{~kb}$ probe labeled in spectrum green was specific for the telomeric side of $E W S$ gene and the $500 \mathrm{~kb}$ probe labeled in spectrum red was specific for the centromeric side of EWS gene. The result of FISH showed the cells with the rearrangement of EWS gene had splits between green signal and red one (Fig. 3). Because the CCS frequently have EWS gene rearrangement (frequency is reported as up to $93 \%$ [3]) and MM usually do not have that, we diagnosed this case as CCS. Systemic screening with CT and MRI revealed two lesions-one in the second lumbar vertebra ( $8 \mathrm{~mm}$ in longest diameter) and the other in the ilium (16 $\mathrm{mm}$ at the longest diameter). Ilium bone biopsy revealed that the iliac lesion had the same immunohistochemical characteristics as the mediastinal tumor. Because the mediastinal tumor turned out to be the largest and CCS originating in those bones is relatively rare, we inferred that the anterior mediastinum was the primary CCS site. Curative resection is not feasible with multiple distant metastases. Chemotherapy with cisplatin $\left(120 \mathrm{mg} / \mathrm{m}^{2}\right)$ and adriamycin $\left(30 \mathrm{mg} / \mathrm{m}^{2}\right)$ was administrated to the patient.

\section{Discussion}

Sarcomas originating in the mediastinum are rare and reportedly account for only $1 \%$ of all referred sarcoma cases [1]. CCS is a rare soft tissue sarcoma, which usually occurs in the tendons and aponeuroses in the extremities of young adults aged 15-40 years. It is often misdiagnosed as MM because it shares similar immunophenotypes. However, recent studies in molecular pathology showed that most CCS cases can be successfully distinguished from $\mathrm{MM}$ on the basis of $E W S$ gene rearrangement caused by the chromosomal translocation $\mathrm{t}(12 ; 22)(\mathrm{q} 13 ; \mathrm{q} 12)$ [2], as seen in our patient. There are several reports showing an ectopic origin of CCS, such as in the gastrointestinal viscera, of which only one case was a primary CCS of the mediastinum [3].

There is no standard treatment for CCS. For resectable cases, extended surgery followed by adjuvant chemotherapy and/or radiotherapy is recommended. Unfortunately, this patient had distant metastases in multiple bones; therefore, chemotherapy was the only choice at that point. Adriamycin, ifosfamide, cisplatin, and vincristine are chemotherapeutic agents commonly used for metastatic CCS. On the other hand, dacarbazine is a key drug for metastatic MM. Recent research has shown that a 

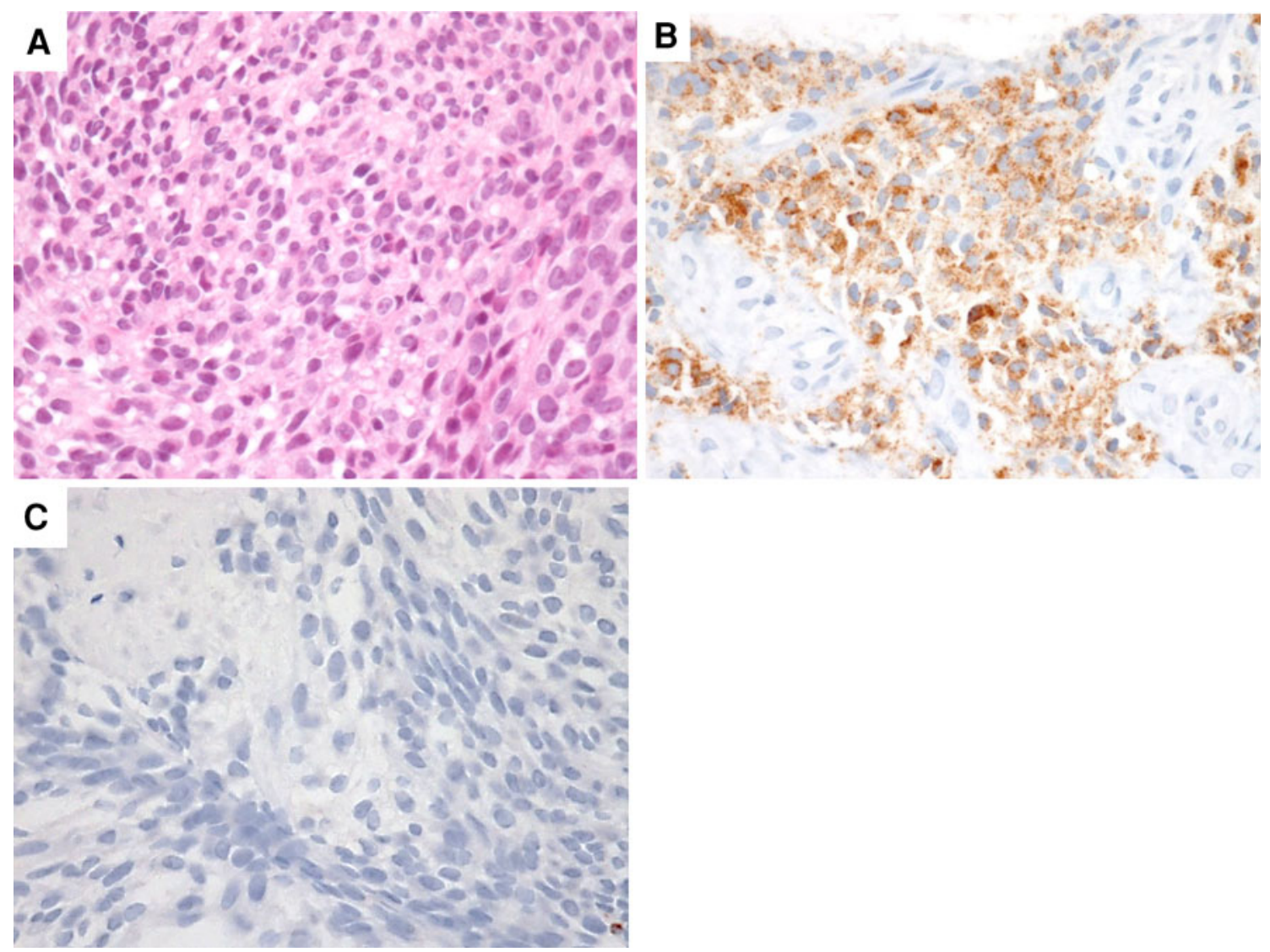

Fig. 2 Microscopic findings of the anterior mediastinal tumor. a Clusters of atypical cells with prominent nucleoli (hematoxylin-eosin staining). b Immunohistochemistry showing tumor cells diffusely positive for HMB-45. c Immunohistochemistry revealed negative for Melan-A

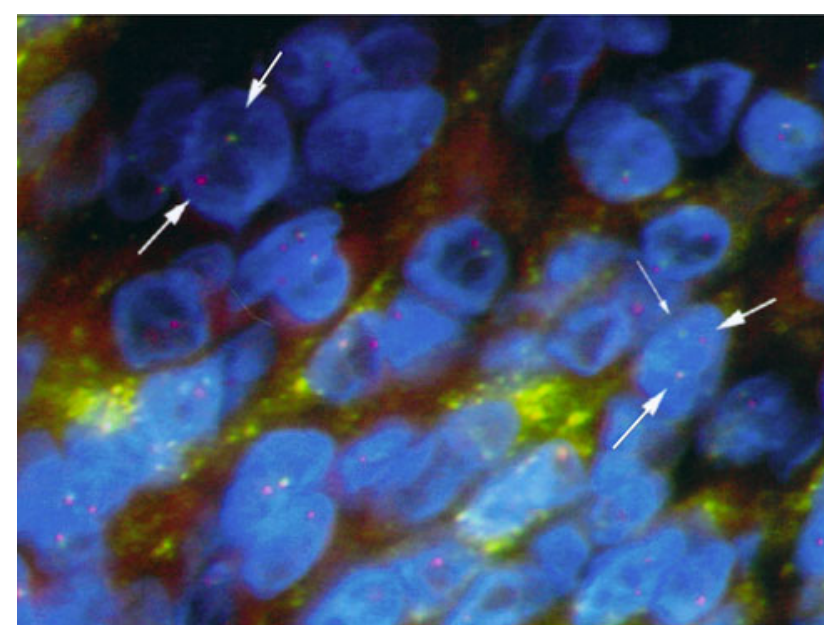

Fig. 3 Fluorescence in situ hybridization results showing tumor cells with break-apart signals (arrows), indicating EWS gene rearrangement (Vysis, LSI EWSR1 Break Apart FISH Probe Kit)

combination of ipilimumab with chemotherapy and BRAF inhibitors are promising alternatives for MM treatment. Thus, differential diagnoses will become more important in the near future because treatment strategies will differ between metastatic CCS and MM. Meticulous observation of skin lesions and pathological and molecular diagnoses, including EWS gene rearrangement, is recommended in cases with mediastinal tumors suspected as MM or CCS.

Although this disease is rare, recent advancements in diagnostics have contributed to accumulating cases to clarify the clinical characteristics of the disease and to develop future molecularly targeted therapies such as PDGFR inhibitor [4] and MET inhibitor [5]. The melanocyte master transcriptional factor (MITF) is reported as a direct transcriptional target of EWS-ATF1 [5]. MITF directly activates the c-Met gene. Because of that, MET inhibitor is thought to be one of the promising agents in CCS.

In conclusion, we experienced a case of CCS, originating in the anterior mediastinum, in which the EWS gene rearrangement test was useful for differential diagnosis with MM.

Conflict of interest The authors declare no conflict of interest.

\section{References}

1. Paquette M, Truong PT, Hart J et al (2010) Primary sarcoma of the mediastinum. A report of 16 cases referred to the British Columbia Cancer Agency. J Thorac Oncol 5:898-906 
2. Zucman J, Delattre O, Desmaze C et al (1993) EWS and ATF-1 gene fusion induced by $\mathrm{t}(12 ; 22)$ translocation in malignant melanoma of soft parts. Nat Genet 4:341-345

3. Tirabosco R, Lang-Lazdunski L, Diss TC, et al. (2009) Clear cell sarcoma of the mediastinum. Ann Diagn Pathol 13:197-200

4. Stacchiotti S, Grosso F, Negri T et al (2010) Tumor response to sunitinib malate observed in clear-cell sarcoma. Ann Oncol 21:1130-1131
5. Davis IJ, McFadden AW, Zhang Y et al (2010) Identification of the receptor tyrosine kinase c-Met and its ligand, hepatocyte growth factor, as therapeutic targets in clear-cell sarcoma. Cancer Res 70:639-645 